




\title{
Dictatorship versus manipulability
}

\author{
Dezső Bednay, ${ }^{(1)}$ Anna Moskalenko, ${ }^{(2)}$ and Attila Tasnádi ${ }^{(1)}$
}

(1) Department of Mathematics, Corvinus University of Budapest, 1093 Budapest, Fővám tér 8 , Hungary

(2) Department of Economics, Universitat Rovira i Virgili and CREIP, Avinguda de la Universitat 1, Reus, Spain

December 2018

\begin{abstract}
The Gibbard-Satterthwaite (1973/75) theorem roughly states that we have to accept dictatorship or manipulability in case of at least three alternatives. A large strand of the literature estimates the degree of manipulability of social choice functions (e.g. Aleskerov and Kurbanov, 1999, Favardin et al., 2002, and Aleskerov et al., 2012), most of them employing the Nitzan-Kelly index of manipulability. We take a different approach and introduce a non-dictatorship index based on our recent work (Bednay et al., 2017), where we have analysed social choice functions based on their distances to the dictatorial rules. By employing computer simulations, we investigate the relationship between the manipulability and nondictatorship indices of some prominent social choice functions, putting them into a common framework.
\end{abstract}

Keywords: Voting rules, dictatorship, manipulability, manipulability index, dictatorship index.

JEL Classification Number: D71.

\section{Introduction}

The classic result of Gibbard (1973) and Satterthwaite (1975) states that for at least three alternatives every universal and resolute social choice function is either manipulable or dictatorial. There is a large literature on how to escape from the negative implications of the Gibbard-Satterthwaite theorem by restricting the set of possible preference profiles, most of them related to single-peaked preferences and their generalizations (e.g. Black, 1958; Moulin, 1980; Barbera et al., 1993; and Puppe and Nehring, 2007a and 2007b, just to name a few). Since the normative approach does not give us the ultimate answer for choosing between social choice functions, another strand of the literature tries to estimate to what extent different voting rules are susceptible to manipulation and to compare the common voting rules according to their 'degree of manipulability'.

There is no universally accepted way to measure the degree of manipulability, but one of the most common approaches is to consider the ratio of preference profiles where manipulation is possible to the total number of profiles, which is called the Nitzan-Kelly's index (NKI, hereinafter) of manipulability, since it was first introduced in Nitzan (1985) and Kelly (1988) 1 A voting rule is thought to be less manipulable if it is manipulable at fewer pref-

\footnotetext{
${ }^{1}$ For other indices of manipulability, see Smith (1999).
} 
erence profiles, or equivalently, if it has a smaller NKI (clearly, the dictatorial voting rule is the least manipulable one). There are a number of studies investigating voting rules under this approach. Kelly (1988) found the minimal number of manipulable profiles for social choice rules which are unanimous and non-dictatorial. This research direction is continued in Fristrup and Keiding (1988) and a series of studies in Maus et al. (2007a, 2007b, 2007c, 2007d).

Kelly (1993) compares the manipulability of the Borda rule with the manipulability of different classes of social choice procedures by developing computational results. Aleskerov and Kurbanov (1999) continue this line of research. The authors study the degree of manipulability of several social choice rules via computational experiments, considering the NKI and in addition introducing some new indices, which are further elaborated in Aleskerov et al. (2009, 2011, 2012). Peters et al. (2012) study both theoretically and using simulations the manipulability of approval voting rule and a family of $k$-approval rules.

In this paper we follow a different route and formulate indices in relation to the dictatorial voting rule, thus picking dictatorship as a reference point instead of manipulability, when looking at the two incompatible properties appearing in the Gibbard-Satterthwaite theorem. In Bednay, Moskalenko and Tasnádi (2017) we have derived the plurality rule as the most balanced one in the sense that it minimizes the sum of the distances to all dictatorial rules, and we have obtained the reverse-plurality rule by maximizing the distance to the closest dictatorial rule. Based on this approach we introduce the non-dictatorship index (NDI). When employing manipulability indices on the set of commonly used social choice functions, the literature strives for the rules with the lowest manipulability index by assuming that a relatively less manipulable rule is deemed to be more desirable. In an analogous way, we are looking for the social choice function with the highest NDI, i.e., the social choice function with low degree of dictatorship.

Our research is also motivated by the fact that regarding manipulability and nondictatorship there is a kind of weak agreement on the 'most extreme' social choice functions. In particular, the reverse-plurality rule, which is the most extreme social choice function in the sense that it lies the furthest away from the closest dictatorial rule, is also group manipulable at every preference profile. Moreover, the reverse-dictatorial social choice function, which always chooses the worst alternative of a fixed voter, is individually manipulable at each profile. Trivially, any dictatorial voting rule is non-manipulable at any preference profile.

The aim of this paper is to investigate the relationship between NDIs and NKIs and to put them into a common framework. By employing computer simulations, we estimate the NDIs of some well-known social choice functions (some scoring rules and Condorcet consistent rules). We calculate NDIs for 3,4 and 5 alternatives and up to 100 voters by generating 1000 random preference profiles, where each profile is selected with the same probability, i.e. we assume an impartial culture. We find that among the prominent social choice functions the plurality rule has the smallest NDI, the Borda count, the Black rule and the Copeland method follow with approximately identical NDIs, while $k$-approval voting (for $k=2$ or $k=3$ ) has the highest NDI among the most common social choice functions. In measuring manipulability we restrict ourselves to NKI, which measures the strategyproofness by counting the number of profiles on which a social choice function is manipulable. While for determining the values for NDI we have written our own program, for determining NKI we have downloaded the results available at Aleskerov et al. (2013) ${ }^{2}$ where we employ the alphabetical tie-breaking rule. Thereafter, we compare our results with Aleskerov et al (2011).

We find that, when unifying the NDIs and NKIs for our social choice functions under

\footnotetext{
${ }^{2}$ http://manip.hse.ru/index.html
} 
study, both indices move in the opposite directions, which is a plausible sign for our nondictatorship index. Next we look at both NDI and NKI of the social choice functions. From our findings we would like to highlight that basically the plurality rule performs the worst in terms of both NDI and NKI with the exception of the case of 4 alternatives for which the 3 -approval voting rule has an even higher NKI. However, there is no such analogously best performing rule based on the two indices.

The structure of the paper is as follows. Section 2 introduces the basic notations and the indices to measure the degree of dictatorship of social choice functions. Section 3 presents the social choice rules under study. Section 4 explains the computational scheme, presents and discusses the results. Finally, Section 5 concludes.

\section{The framework}

Let $A=\{1, \ldots, m\}$ be the set of alternatives, where $m \geq 2$, and $N=\{1, \ldots, n\}$ be the set of voters. We shall denote by $\mathcal{P}$ the set of all linear orderings (irreflexive, transitive and total binary relations) on $A$ and by $\mathcal{P}^{n}$ the set of all preference profiles. If $\succ \in \mathcal{P}^{n}$ and $i \in N$, then $\succ_{i}$ is the preference ordering of voter $i$ over $A$.

Definition 1. A mapping $f: \mathcal{P}^{n} \rightarrow A$ that selects the winning alternative is called a social choice function, henceforth, SCF.

As our definition of an SCF does not allow for possible ties, in this event a fixed (anonymous) tie-breaking rule will be employed. A tie-breaking rule $\tau: \mathcal{P}^{n} \rightarrow \mathcal{P}$ maps preference profiles to linear orderings on $A$, which will be only employed when a formula does not determine a unique winner. If there are more alternatives chosen by a formula 'almost' specifying an SCF, then the highest ranked alternative is selected, based on the given tie-breaking rule among tied alternatives. When determining the NKIs for the voting rules under study, we employ the lexicographic tie-breaking rule $1 \succ 2 \succ \cdots \succ m$, which is anonymous and does not depend on the actual preference profile $\succ \in \mathcal{P}^{n}$.

Let $\mathcal{F}=A^{\mathcal{P}^{n}}$ be the set of SCFs and $\mathcal{F}^{a n} \subset \mathcal{F}$ be the set of anonymous voting rules. The subset of $\mathcal{F}$ consisting of the dictatorial rules will be denoted by $\mathcal{D}=\left\{d_{1}, \ldots, d_{n}\right\}$, where $d_{i}$ is the dictatorial rule with voter $i$ as the dictator. By counting the number of profiles, on which $f$ and $g$ choose different alternatives we define a metric:

$$
\rho(f, g)=\#\left\{\succ \in \mathcal{P}^{n} \mid f(\succ) \neq g(\succ)\right\}
$$

on $\mathcal{F}=A^{\mathcal{P}^{n}}$.

We define our non-dictatorship index (NDI) by taking the distance to the closest dictator.

Definition 2. The non-dictatorship index (NDI) is given by

$$
N D I(f)=\min _{i \in N} \rho\left(f, d_{i}\right)
$$

We specify the set of least dictatorial rules by those ones which are the furthest away from the closest dictatorial rule, which means that we are maximizing the minimum of the distances to the dictators.

Definition 3. We define the set of least dictatorial rules by

$$
\mathcal{F}_{l d}=\arg \max _{f \in \mathcal{F}} \min _{i \in N} \rho\left(f, d_{i}\right)=\arg \max _{f \in \mathcal{F}} N D I(f)
$$

in general and by

$$
\mathcal{F}_{l d}^{a n}=\arg \max _{f \in \mathcal{F}^{a n}} \min _{i \in N} \rho\left(f, d_{i}\right)=\arg \max _{f \in \mathcal{F}^{a n}} N D I(f)
$$


over the set of anonymous voting rules.

In Bednay, Moskalenko and Tasnádi (2017) we have established that $\mathcal{F}_{l d}^{a n}$ equals the set of reverse-plurality rules with anonymous tie-breaking rules, where the reverse-plurality rule $f_{\tau}^{*}$ selects the alternative being the fewest times on the top and in case of ties, a fixed anonymous tie-breaking rule $\tau$ is employed.

\section{$3 \quad$ Voting rules}

We will need some additional notations. Let $q$ be the cardinality of $A$ and let $s:\{1, \ldots, q\} \rightarrow$ $\mathbf{R}$ satisfy $s(1) \geq s(2) \geq \ldots \geq s(q)$ and $s(1)>s(q)$. Moreover, let $r k[a, \succ]$ denote the rank of alternative $a$ in the ordering $\succ \in \mathcal{P}$ (i.e. $r k[a, \succ]=1$ if $a$ is the top alternative in the ranking $\succ, r k[a, \succ]=2$ if $a$ is second-best, and so on). We consider the following five common voting rules.

1. Plurality Rule: A voting rule $P L$ is the plurality rule if for all $\left(\succ_{i}\right)_{i=1}^{n} \in \mathcal{P}^{n}$

$$
P L\left(\left(\succ_{i}\right)_{i=1}^{n}\right)=\arg \max _{a \in A} \#\left\{i \in N \mid r k\left[a, \succ_{i}\right]=1\right\} .
$$

$P L$ choose the alternatives that are ranked first by the maximum number of voters.

2. Borda Count: We shall denote the Borda score of alternative $a \in A$ according to ordering $\succ$ by $b s[a, \succ]=q-r k[a, \succ]$. A voting rule $B C$ is the Borda count if for all $\left(\succ_{i}\right)_{i=1}^{n} \in \mathcal{P}^{n}$

$$
B C\left(\left(\succ_{i}\right)_{i=1}^{n}\right)=\arg \max _{a \in A} \sum_{i=1}^{n} b s\left[a, \succ_{i}\right] .
$$

$B C$ choose the alternatives with the maximum Borda score $b s$. $\mathcal{P}^{n}$

3. $k$-Approval Rule: A voting rule $k-A V$ is the $k$-approval voting rule if for all $\left(\succ_{i}\right)_{i=1}^{n} \in$

$$
k-A V\left(\left(\succ_{i}\right)_{i=1}^{n}\right)=\arg \max _{a \in A} \#\left\{i \in N \mid r k\left[a, \succ_{i}\right] \leq k\right\} .
$$

$k-A V$ chooses the alternatives which are admitted to be among the $k$ best by the highest number of voters. We will consider $k-A V$ for $k=2,3$.

4. Copeland Method: For a given profile $\left(\succ_{i}\right)_{i=1}^{n} \in \mathcal{P}^{n}$ we say that alternative $a \in A$ beats alternative $x \in A$ if $\#\left\{i \in N \mid a \succ_{i} x\right\}>\#\left\{i \in N \mid x \succ_{i} a\right\}$, i.e. $a$ wins over $x$ by pairwise comparison. We shall denote by $l\left[a,\left(\succ_{i}\right)_{i=1}^{n}\right]$ the number of alternatives beaten by alternative $a \in A$ for a given profile $\left(\succ_{i}\right)_{i=1}^{n}$. Then a voting rule $C M$ is the Copeland method if for all $\left(\succ_{i}\right)_{i=1}^{n} \in \mathcal{P}^{n}$

$$
C M\left(\left(\succ_{i}\right)_{i=1}^{n}\right)=\arg \max _{a \in A} l\left[a,\left(\succ_{i}\right)_{i=1}^{n}\right] .
$$

5. Black's procedure: Let $\mu$ be a majority relation for a given profile $\left(\succ_{i}\right)_{i=1}^{n} \in \mathcal{P}^{n}$, then $a \mu x$ if $\#\left\{i \in N \mid a \succ_{i} x\right\}>\#\left\{i \in N \mid x \succ_{i} a\right\}$. The Condorcet winner $C W$ in a profile $\left(\succ_{i}\right)_{i=1}^{n}$ is an element beating any other alternative based on the majority relation $\mu$ (constructed according to the profile), i.e.

$$
C W\left(\left(\succ_{i}\right)_{i=1}^{n}\right)=\{a \in A \mid \text { for all } x \in A \backslash\{a\}: a \mu x\} .
$$

Black's rule chooses the Condorcet winner if it exists, otherwise it chooses the alternative with the highest Borda score. 


\section{Computation scheme and results}

The calculation of indices is performed for 3, 4 and 5 alternatives and up to 100 voters. We generate 1000 random preference profiles, where each profile occurs with equal probability, i.e. under the Impartial Culture assumption.

Tables 1, 2 and 3 present the results of the NDIs of the voting rules for the case of 3 , 4 and 5 alternatives and for 3, 4, 5, 20 and 100 voters. We look at the non-dictatorship property of the 6 voting rules, taking into account the reverse-plurality rule, which serves as a benchmark (clearly, it has the highest NDI). It can be readily seen that among the common voting rules the plurality rule performs the worst, having the lowest NDI. The $k$ - approval voting $(k=3$ or $k=2)$ performs the best, with the highest NDI. The Borda count, Black's rule and the Copeland method lie between the plurality and the $k$-approval rules without any clear difference between them.

\begin{tabular}{|c|c|c|c|c|c|c|}
\hline \multirow{2}{*}{ Voting rules } & \multicolumn{5}{|c|}{ Number of voters } \\
\cline { 2 - 7 } & 3 & 4 & 5 & 10 & 20 & 100 \\
\hline Plurality & 0.360 & 0.392 & 0.427 & 0.484 & 0.533 & 0.583 \\
Borda & 0.363 & 0.442 & 0.450 & 0.486 & 0.527 & 0.587 \\
$k$-Approval $k=2$ & 0.488 & 0.550 & 0.544 & 0.571 & 0.570 & 0.605 \\
$k$-Approval $k=3$ & 0.642 & 0.661 & 0.656 & 0.648 & 0.644 & 0.627 \\
Copeland & 0.348 & 0.438 & 0.439 & 0.491 & 0.527 & 0.581 \\
Black & 0.348 & 0.442 & 0.436 & 0.486 & 0.519 & 0.585 \\
Reverse-plurality & 0.918 & 0.881 & 0.867 & 0.797 & 0.746 & 0.682 \\
\hline
\end{tabular}

Table 1: Non-dictatorship indices in case of three alternatives

\begin{tabular}{|c|c|c|c|c|c|c|}
\hline \multirow{2}{*}{ Voting rules } & \multicolumn{5}{|c|}{ Number of voters } \\
\cline { 2 - 7 } & 3 & 4 & 5 & 10 & 20 & 100 \\
\hline Plurality & 0.437 & 0.448 & 0.485 & 0.548 & 0.602 & 0.655 \\
Borda & 0.455 & 0.501 & 0.531 & 0.575 & 0.628 & 0.677 \\
$k$-Approval $k=2$ & 0.591 & 0.580 & 0.613 & 0.629 & 0.649 & 0.688 \\
$k$-Approval $k=3$ & 0.617 & 0.662 & 0.669 & 0.695 & 0.685 & 0.701 \\
Copeland & 0.437 & 0.493 & 0.506 & 0.565 & 0.629 & 0.673 \\
Black & 0.435 & 0.499 & 0.510 & 0.571 & 0.628 & 0.676 \\
Reverse-plurality & 1 & 0.969 & 0.950 & 0.882 & 0.842 & 0.770 \\
\hline
\end{tabular}

Table 2: Non-dictatorship indices in case of four alternatives

\begin{tabular}{|c|c|c|c|c|c|c|}
\hline \multirow{2}{*}{ Voting rules } & \multicolumn{7}{|c|}{ Number of voters } \\
\cline { 2 - 6 } & 3 & 4 & 5 & 15 & 20 & 100 \\
\hline Plurality & 0.449 & 0.497 & 0.524 & 0.618 & 0.651 & 0.706 \\
Borda & 0.517 & 0.556 & 0.581 & 0.673 & 0.685 & 0.735 \\
$k$-Approval $k=2$ & 0.601 & 0.623 & 0.641 & 0.699 & 0.705 & 0.725 \\
$k$-Approval $k=3$ & 0.676 & 0.692 & 0.704 & 0.733 & 0.716 & 0.744 \\
Copeland & 0.465 & 0.552 & 0.567 & 0.660 & 0.684 & 0.727 \\
Black & 0.473 & 0.554 & 0.572 & 0.662 & 0.684 & 0.734 \\
Reverse-plurality & 1 & 1 & 0.991 & 0.906 & 0.898 & 0.824 \\
\hline
\end{tabular}

Table 3: Non-dictatorship indices in case of five alternatives

The graphical representations of the NDIs for 3,4 and 5 alternatives case and up to 100 voters are shown in Figures 1, 22 and 3. On the X-axis we have the number of voters and on the Y-axis we have the values of NDIs. From these figures we observe that the 
reverse-plurality rule has the highest NDI, while the plurality rule has the lowest NDI. The NDIs of the other voting rules under study lie between the NDIs of these two rules.

$\mathrm{NDI}(m=3)$

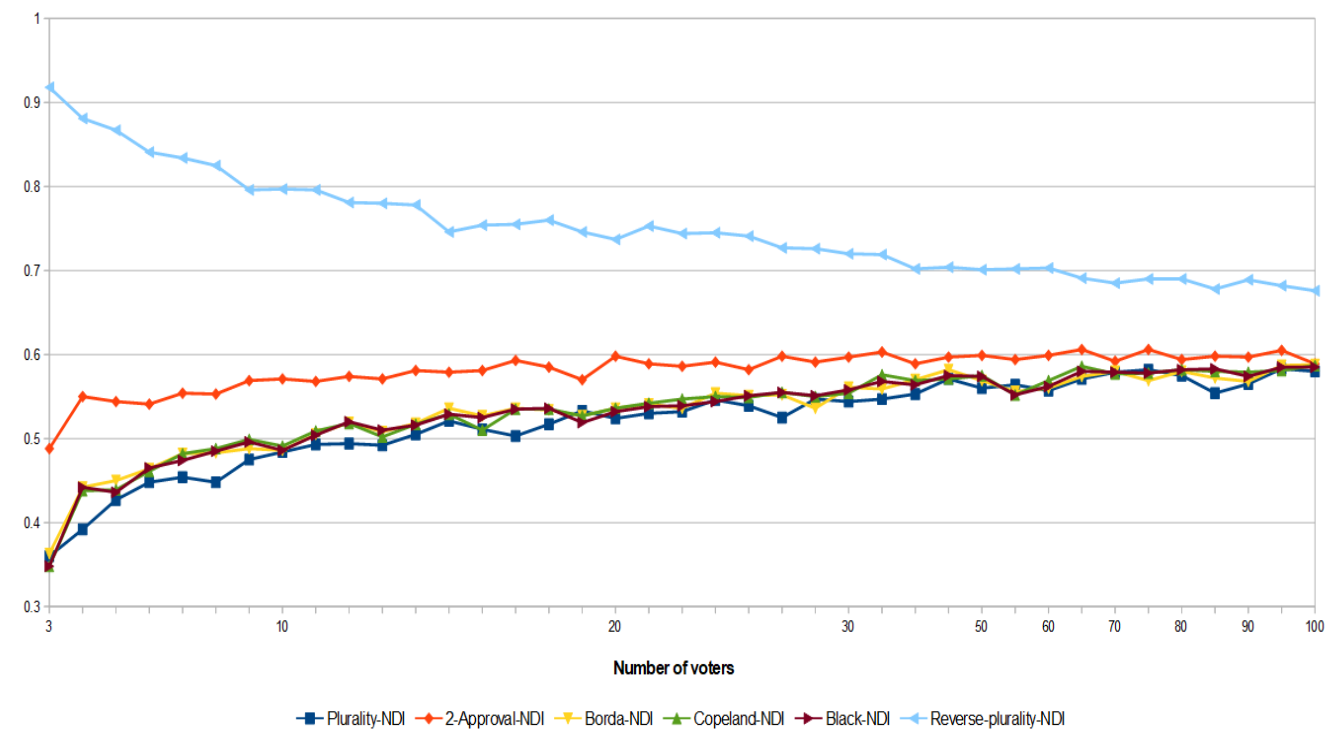

Figure 1: Non-dictatorship indices in case of three alternatives



Figure 2: Non-dictatorship indices in case of four alternatives 


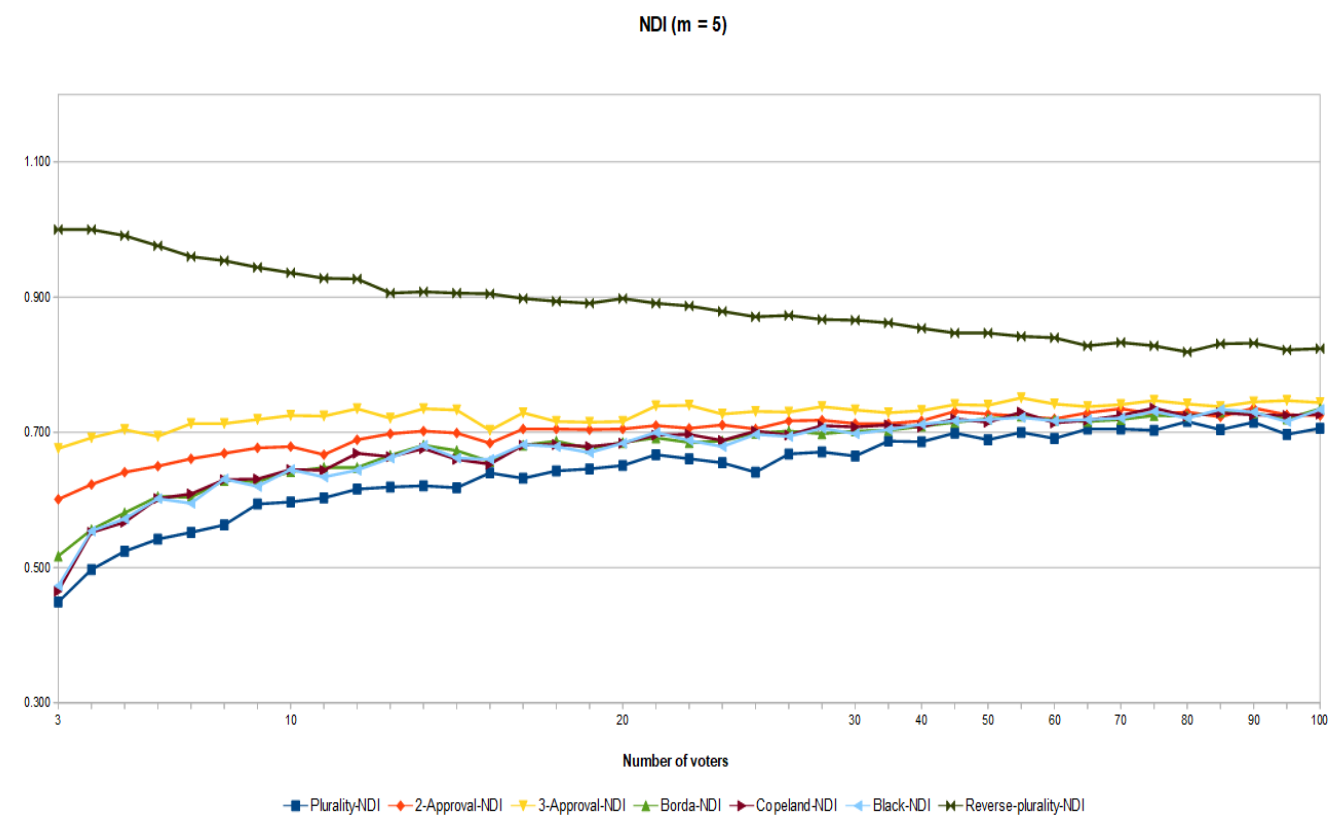

Figure 3: Non-dictatorship indices in case of five alternatives

If we also add the degree of manipulability, we expect that while the NDIs of common voting rules are increasing, their NKIs are decreasing in the number of voters. To check this, we put both the NDIs and NKIs in the same tables and figures. Tables 4 , 5 and 6 show the unified results for the NKIs and NDIs of the voting rules under study for the case of 3 , 4 and 5 alternatives, and 3, 4 and 10 voters. High NDI means a low degree of dictatorship, while low NKI means a low degree of manipulability. Thus, the voting rule which has high NDI and low NKI will perform the best.

\begin{tabular}{|c|c|c|c|c|c|c|c|c|}
\hline \multirow{2}{*}{ Voting rules } & \multicolumn{2}{|c|}{$n=3$} & \multicolumn{2}{c|}{$n=4$} & \multicolumn{2}{c|}{$n=10$} & \multicolumn{2}{c|}{$n=100$} \\
\cline { 2 - 8 } & NDI & NKI & NDI & NKI & NDI & NKI & NDI & NKI \\
\hline Plurality & 0.360 & 0.167 & 0.392 & 0.185 & 0.484 & 0.284 & 0.583 & 0.139 \\
Borda & 0.363 & 0.236 & 0.442 & 0.310 & 0.486 & 0.241 & 0.587 & 0.083 \\
$k$-Approval $k=2$ & 0.488 & 0.264 & 0.550 & 0.275 & 0.571 & 0.278 & 0.605 & 0.128 \\
Copeland & 0.348 & 0.111 & 0.438 & 0.296 & 0.491 & 0.194 & 0.581 & 0.056 \\
Black & 0.348 & 0.111 & 0.442 & 0.144 & 0.486 & 0.147 & 0.585 & 0.065 \\
\hline
\end{tabular}

Table 4: NDIs and NKIs for the case of three alternatives

\begin{tabular}{|c|c|c|c|c|c|c|c|c|}
\hline \multirow{2}{*}{ Voting rules } & \multicolumn{2}{|c|}{$n=3$} & \multicolumn{2}{c|}{$n=4$} & \multicolumn{2}{c|}{$n=10$} & \multicolumn{2}{c|}{$n=100$} \\
\cline { 2 - 8 } & NDI & NKI & NDI & NKI & NDI & NKI & NDI & NKI \\
\hline Plurality & 0.437 & 0.294 & 0.448 & 0.325 & 0.548 & 0.421 & 0.655 & 0.187 \\
Borda & 0.455 & 0.512 & 0.501 & 0.500 & 0.575 & 0.419 & 0.677 & 0.153 \\
$k$-Approval $k=2$ & 0.591 & 0.394 & 0.580 & 0.426 & 0.629 & 0.419 & 0.688 & 0.170 \\
$k$-Approval $k=3$ & 0.617 & 0.500 & 0.662 & 0.525 & 0.695 & 0.490 & 0.701 & 0.198 \\
Copeland & 0.437 & 0.227 & 0.493 & 0.453 & 0.565 & 0.343 & 0.673 & 0.135 \\
Black & 0.435 & 0.276 & 0.499 & 0.263 & 0.571 & 0.275 & 0.676 & 0.127 \\
\hline
\end{tabular}

Table 5: NDIs and NKIs for the case of four alternatives 


\begin{tabular}{|c|c|c|c|c|c|c|c|c|}
\hline \multirow{2}{*}{ Voting rules } & \multicolumn{2}{|c|}{$n=3$} & \multicolumn{2}{c|}{$n=4$} & \multicolumn{2}{c|}{$n=15$} & \multicolumn{2}{c|}{$n=100$} \\
\cline { 2 - 8 } & NDI & NKI & NDI & NKI & NDI & NKI & NDI & NKI \\
\hline Plurality & 0.449 & 0.389 & 0.497 & 0.426 & 0.618 & 0.469 & 0.706 & 0.227 \\
Borda & 0.517 & 0.691 & 0.556 & 0.639 & 0.673 & 0.465 & 0.735 & 0.206 \\
$k$-Approval $k=2$ & 0.517 & 0.691 & 0.556 & 0.639 & 0.673 & 0.465 & 0.735 & 0.206 \\
$k$-Approval $k=3$ & 0.676 & 0.576 & 0.692 & 0.582 & 0.733 & 0.464 & 0.744 & 0.201 \\
Copeland & 0.465 & 0.329 & 0.552 & 0.561 & 0.660 & 0.382 & 0.727 & 0.202 \\
Black & 0.473 & 0.409 & 0.554 & 0.357 & 0.662 & 0.419 & 0.734 & 0.182 \\
\hline
\end{tabular}

Table 6: NDIs and NKIs for the case of five alternatives

Figures 4 . 5 and 6 show graphically the results for both NDIs and NKIs. We can see that both NDIs and NKIs are moving in the opposite directions, which is plausible and a positive sign for our non-dictatorship index. In case of three alternatives $(m=3)$, the plurality rule performs the worst from the point of view of both dictatorship and manipulability. However, when there are four alternatives $(m=4)$, the rule that performs the worst from the point of view of manipulability is the $k$-approval rule with $k=3$, and the plurality rule performs the worst from the point of view of dictatorship (has the lowest NDI), while the $k$-approval rule with $k=3$ has the highest NDI, but of course, without taking into account the reverseplurality rule's NDI. Considering the case of five alternatives $(m=5)$, for a small number of voters $(n<15)$, the Borda count has the highest degree of manipulability (NKI is high), while from the point of view of dictatorship its NDI is identical to Black's rule and lies between the plurality and $k$-approval rule with $k=3$. As the number of voters increases $(n \geq 15)$, again the only voting rule that performs the worst from the point of view of both manipulability and dictatorship is the plurality rule (with the exception of the 3-approval voting rule, which, for the case of four alternatives, has a higher NKI).

$\mathrm{NDI}$ and $\mathrm{NKI}(\mathrm{m}=3)$

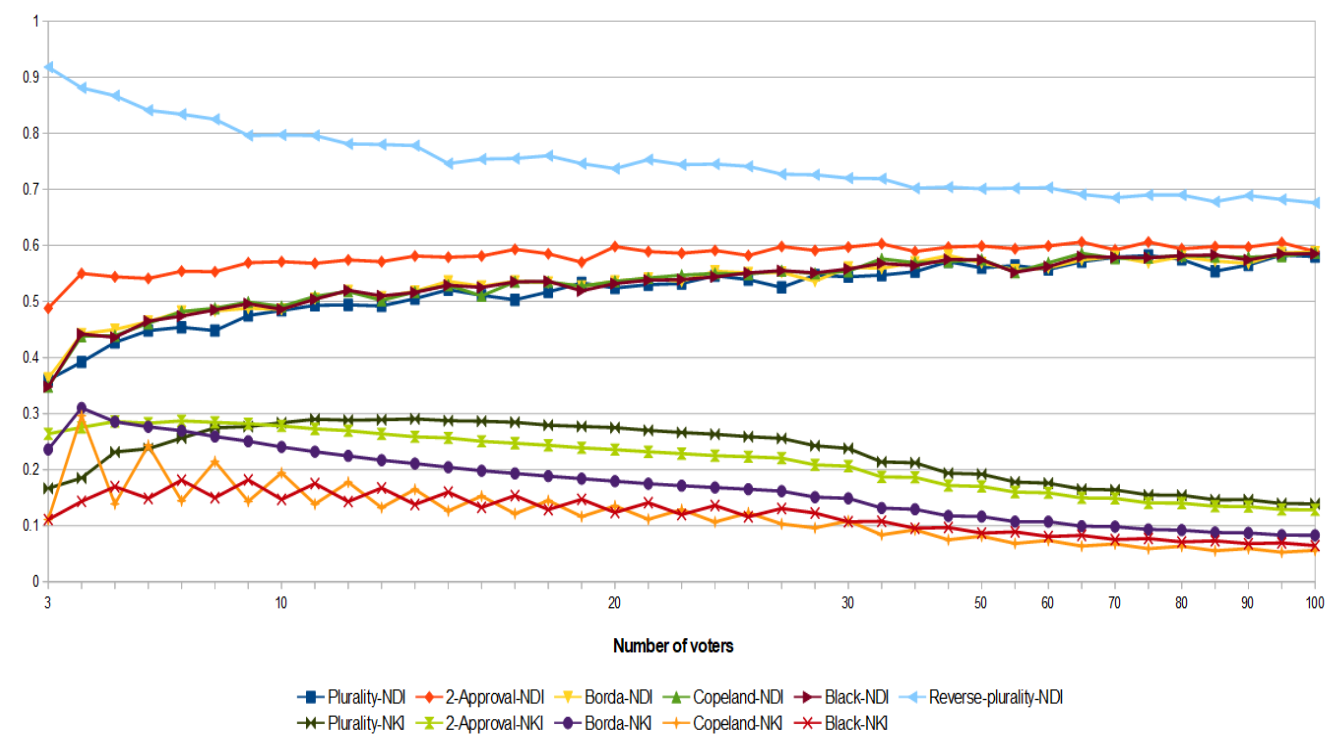

Figure 4: Non-dictatorship and Nitzan-Kelly indices in case of 3 alternatives 
$\mathrm{NDI}$ and $\mathrm{NKI}(\mathrm{m}=4)$

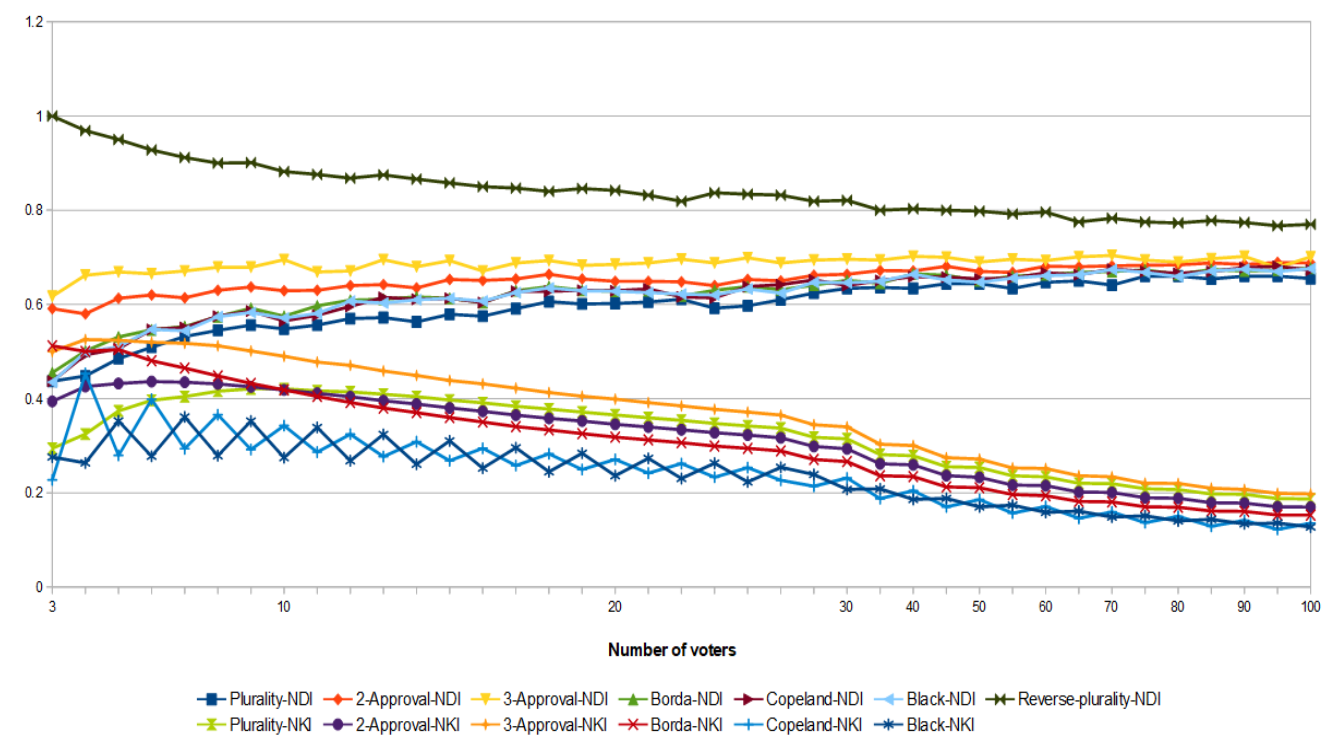

Figure 5: Non-dictatorship and Nitzan-Kelly indices in case of 3 alternatives

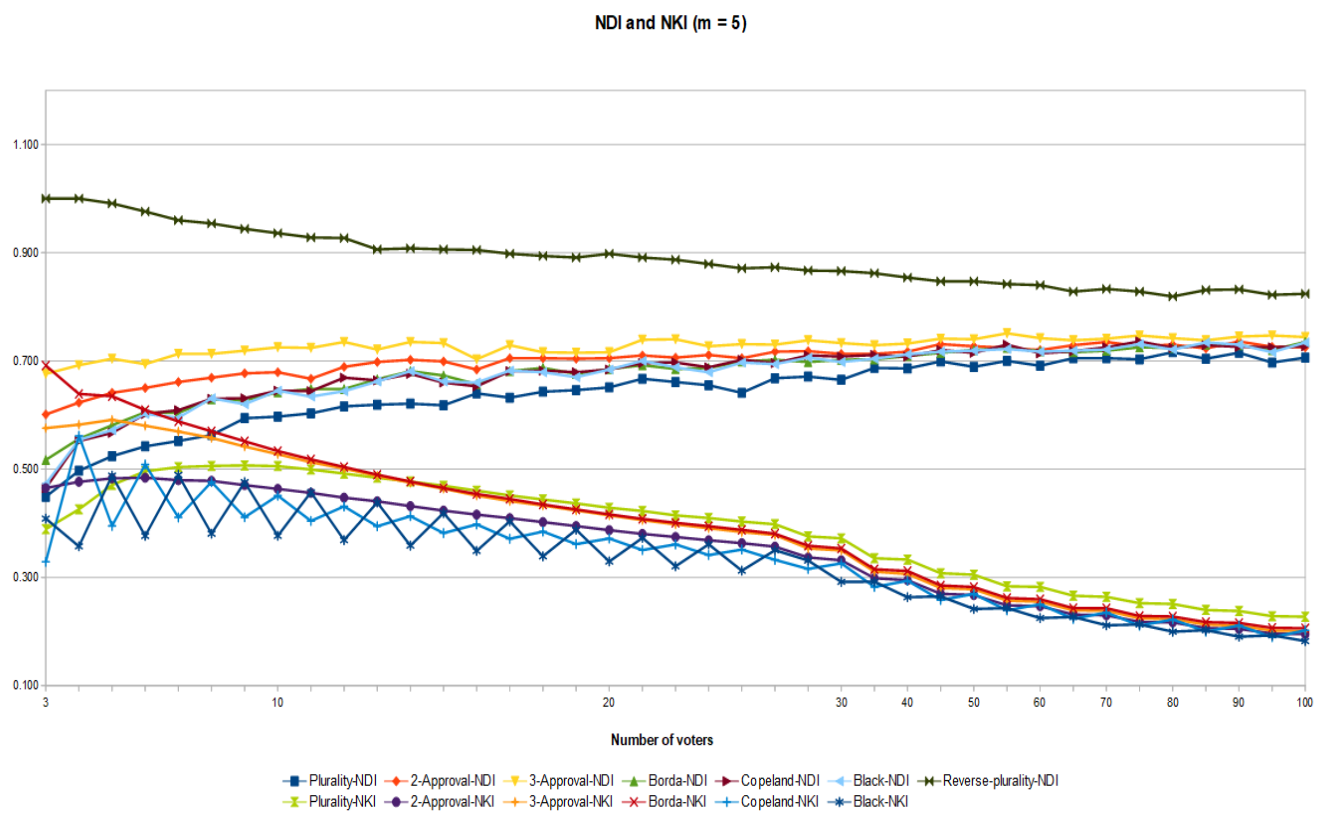

Figure 6: Non-dictatorship and Nitzan-Kelly indices in case of 3 alternatives 


\section{Conclusion}

Based on the classic Gibbard-Satterthwaite's impossibility theorem the properties of strategyproofness and non-dictatorship are incompatible if there are at least three alternatives, any preference profile is possible and the social choice function has to be onto. Therefore, whenever a decision has to be made about the social choice function to be employed, we always face the dilemma of choosing between a degree of dictatorship and a degree of manipulability.

Both incompatible properties are undesirable. However, when picking a voting rule it could be helpful and informative to know about its degree of manipulability and its distance from dictatorship. Concerning non-manipulability indices, we have selected the NitzanKelly index, which counts the number of manipulable profiles in the total number of profiles from the several non-manipulability indices already employed in the literature. We have introduced a non-dictatorship index, which measures the dictatorial component of voting rules, and we have ranked common voting rules based on this index.

Clearly, we could think about different ways of measuring the dictatorial component of a voting rule. We have chosen a fairly straightforward distance based approach for defining our non-dictatorship index. In this paper we have explored the relationship between the Nitzan-Kelly index and our non-dictatorship index. Though these two approaches differ substantially we nevertheless arrived to the same conclusion that among the prominent voting rules basically the plurality rule performs the worst from the point of view of dictatorship and manipulability.

Finally, we would like to mention that since by having less manipulable profiles we are getting closer to the dictatorial rule it is not at all clear whether minimizing the number of manipulable profiles should be the right goal. We have a similar situation in case of our non-dictatorship index since by maximizing our non-dictatorship index we are getting closer to the undesirable reverse-plurality rule. Despite challenging these approaches we believe that they shed some light on the evergreen problem of choosing an appropriate voting rule.

\section{Acknowledgments}

We thank Péter Biró, Dóra Gréta Petróczy and Sonal Yadav for helpful comments and useful conversations. Bednay and Tasnádi gratefully acknowledge the financial support from the Hungarian Scientific Research Fund (OTKA K-112975). This research was supported by the Pallas Athéné Domus Sapientiae Foundation through its PADS Leading Researcher Program.

\section{References}

[1] Aleskerov, F., D. Karabekyan, M.R. Sanver and V. Yakuba (2011), Evaluation of the degree of manipulability of known aggregation procedures under multiple choice, Journal of the New Economic Association, 1, 37-61.

[2] Aleskerov, F., D. Karabekyan, M.R. Sanver and V. Yakuba (2011), An individual manipulability of positional voting rules, SERIEs, 2, 431-446.

[3] Aleskerov, F., D. Karabekyan, M.R. Sanver and V. Yakuba (2012), On the manipulability of voting rules: The case of 4 and 5 alternatives, Mathematical Social Sciences, 64, 67-73.

[4] Aleskerov, F. and E. Kurbanov (1999), Degree of Manipulability of Social Choice Procedures, in: A. Alkan et.al. (eds.), Current Trends in Economics, Springer, Berlin. 
[5] Barberá, S., F. Gul and E. Stacchetti (1993), Generalized Median Voter Schemes and Committees, Journal of Economic Theory, 61, 262-289.

[6] Bednay, D., Moskalenko, A. and Tasnádi, A. (2017), Does avoiding bad voting rules lead to the good ones? Operations Research Letters, 45, 448-451.

[7] Black, D. (1958), The Theory of Committees and Elections, Cambridge University Press, Cambridge.

[8] Favardin, P., D. Lepelley and J. Serais (2002), Borda Rule, Copeland Method and Strategic Manipulation, Review of Economic Design, 7, 213-228.

[9] Gibbard, A. (1973), Manipulation of voting schemes: a general result, Econometrica, 41, 587-601.

[10] Kelly, J. (1988), Minimal manipulability and local strategy-proofness, Social Choice and Welfare, 5, 81-85.

[11] KelLy, J. (1993), Almost all social choice rules are highly manipulable, but few aren't, Social Choice and Welfare, 10, 161-175.

[12] Maus, S., Peters, H. and Storcken, T (2007a), Minimally Manipulable Anonymous Social Choice Functions, Mathematical Social Sciences, 53, 239-254.

[13] Maus, S., Peters, H. and Storcken, T (2007b), Minimal Manipulability: Unanimity and Nondictatorship, Journal of Mathematical Economics, 43, 675-691.

[14] Maus, S., Peters, H. and Storcken, T (2007c), Anonymous Voting and Minimal Manipulability, Journal of Economic Theory, 135, 533-544.

[15] Maus, S., Peters, H. and Storcken, T (2007d), Minimal Manipulability: Anonymity and Unanimity, Social Choice and Welfare, 29, 247-268.

[16] Moulin, H. (1980), On strategy-proofness and single-peakedness, Public Choice, 35, 437-455.

[17] Nehring, K. and Puppe, C. (2007a), Efficient and strategy-proof voting rules: A characterization, Games and Economic Behaviour, 59, 132-163.

[18] Nehring, K. and Puppe, C. (2007b), The structure of strategy-proof social choice - part i: general characterization and possibility results on median spaces, Journal of Economic Theory, 135, 269-305.

[19] Nitzan, S. (1985), The vulnerability of point-voting schemes to preference variation and strategic manipulation, Public Choice, 47, 349-370.

[20] Peters, H., S. Roy and T. Storcken (2012), On the manipulability of approval voting and related scoring rules, Social Choice and Welfare, 39, 399-429.

[21] Satterthwaite, M. (1975), Strategy-proofness and Arrow's conditions: existence and correspondence theorems for voting procedures and social welfare functions, Journal of Economic Theory, 10, 187-217.

[22] Sмith, D.A. (1999), Manipulability measures of common social choice functions, Social Choice and Welfare, 16, 639-661. 